\title{
Vascular protective effects of Astragalus membranaceus and its main constituents in rats with chronic hyperhomocysteinemia
}

\author{
LI-HONG QIU ${ }^{1}$, BI-QI ZHANG ${ }^{1}$, MIAO-JUN LIAN ${ }^{1}$, XIAN-JI XIE ${ }^{2}$ and PENG CHEN ${ }^{1}$ \\ Departments of ${ }^{1}$ Cardiology and ${ }^{2}$ Pharmacy, The First Affiliated Hospital, College of Medicine, Zhejiang University, \\ Hangzhou, Zhejiang 310003, P.R. China
}

Received October 7, 2016; Accepted April 28, 2017

DOI: $10.3892 /$ etm.2017.4739

\begin{abstract}
Hyperhomocysteinemia (HHcy) is an important factor in cardiovascular disease. However, is currently no cure available in western medicine for HHcy-evoked cardiovascular disease. The present study explored the vascular protective effects of Astragalus membranaceus (AM), a traditional Chinese medicine. Rats with HHcy were induced by feeding high-methionine diets and treated with total extract of AM (TEA) and its constituents, including Astragalus saponins (ASP), Astragalus total flavonoids (ATF) and Astragalus polysaccharides (APS). Examination of the rats indicated that TEA and ASP controlled blood pressure and ameliorated HHcy-induced impairment of endothelium-dependent vasorelaxation by increasing the nitric oxide content and nitric oxide synthase activity of the abdominal aorta. Furthermore, they decreased the accumulation of hydrogen peroxide and superoxide anion, and attenuated the inhibition of superoxide dismutase and catalase activities in rats with HHcy. Additionally, TEA and ASP attenuated the HHcy-induced increases of matrix metalloproteinase (MMP)-2 and -9 concentrations. However, similar effects were not observed for ATF and APS. In conclusion, TEA and ASP are beneficial to vascular disease, and their effects may be attributed to protective actions against oxidation, activity of the MMPs and endothelial dysfunction.
\end{abstract}

\section{Introduction}

Cardiovascular disease, which is characterized by dysfunctional conditions of the heart, arteries and veins, remains the most common cause of morbidity and mortality and is

Correspondence to: Professor Peng Chen, Department of Cardiology, The First Affiliated Hospital, College of Medicine, Zhejiang University, 79 Qingchun Street, Hangzhou, Zhejiang 310003, P.R. China

E-mail: pengchen_zyyy@hotmail.com

Key words: Astragalus membranaceus, homocysteine, oxidation, matrix metalloproteinases, endothelial dysfunction a serious threat to human health (1). Age, gender, cigarette smoking, physical inactivity, high blood pressure and serum cholesterol, elevated serum glucose, obesity and family history have been identified as classic risk factors for the development of cardiovascular disease (2). However, even when all these risk factors are considered, they only explain approximately one-half to two-thirds of the occurrence of cardiovascular disease (1). It appears that additional crucial risk factors remain to be identified. Elevated plasma levels of homocysteine (Hcy) have received considerable interest, as they may predict future cardiovascular disease (3).

Hcy is the main metabolite of methionine, and its levels can be inordinately increased due to genetic enzymatic deficiencies and nutritional defects, both of which affect the proper metabolism of methionine (4). Clinical and animal studies have identified that hyperhomocysteinemia (HHcy) may be important in the damage of the vascular structure and function (5-7). Although a unifying hypothesis has not been established thus far, stress, endothelial dysfunction and vascular remodeling are considered as the primary pathological mechanisms underlying vascular diseases following chronic exposure to Hcy (1). However, in a review of several randomized controlled trials, it was shown that Hcy-lowering interventions were not able to prevent cardiovascular events (8). It appears that there is not yet a significant cure for HHcy-evoked vascular disease in western medicine.

According to the original recording in Shen Nong's Materia Medica >2,000 years ago (9), Astragalus membranaceus (AM) has been widely used in traditional Chinese medicine to treat patients with cardiovascular disease. Astragalus saponins (ASP), Astragalus total flavonoids (ATF) and Astragalus polysaccharides (APS) have been identified as the main bioactive chemicals in AM (9). Observations in previous studies revealed that this herb was able to prevent lipid peroxidation by improving antioxidant enzyme activity and scavenging free radicals, improve vasorelaxation by increasing levels of nitric oxide (NO) and cyclic guanosine monophosphate (cGMP), and hinder the process of pathological vascular remodeling (10-12). Furthermore, previous in vitro studies from the present author's laboratory demonstrated that AM and its main components may be advantageous in the treatment of Hcy-mediated endothelial dysfunction by stabilizing the NO/NO synthase (NOS) pathway $(13,14)$. 
The aim of the present study was to investigate the vascular protective effect of AM and its main constituents in rats with chronic HHcy and to explore the underlying mechanism.

\section{Materials and methods}

Materials. AM roots were collected at Longxi (Gansu, China). A total extract of AM (TEA; yield, 9.8\%) and bioactive constituents (yield: APS, 6.4\%; ATF, 3.6\%, ASP, 1.1\%) from the AM roots was prepared by the Department of Pharmacy at the First Affiliated Hospital, College of Medicine, Zhejiang University (Hangzhou, China) as previously described (14).

Animals and experimental protocol. A total of 72 male 8-week-old Sprague-Dawley rats (weighing 240-260 g) were obtained from Laboratory Animal Center in the Chinese Academy of Science (Shanghai, China). Rats were housed in a room at a constant temperature $\left(23-26^{\circ} \mathrm{C}\right)$ and humidity (40-60\%) with a 12-h light/dark cycle. They were randomly divided into 6 groups (each $\mathrm{n}=12$ ): i) Control, ii) HHcy, iii) TEA, iv) ASP, v) APS and vi) ATF groups. HHcy was induced in the rats by feeding a high-methionine ( $2 \%$ by weight) diet (15). Rats in the control group received a regular diet. In the TEA, ASP, APS and ATF groups, rats fed a high-methionine diet were treated with TEA $(196 \mathrm{mg} / \mathrm{kg})$, APS $(128 \mathrm{mg} / \mathrm{kg}), \operatorname{ATF}(72 \mathrm{mg} / \mathrm{kg})$ and ASP $(22 \mathrm{mg} / \mathrm{kg})$, respectively, by intraperitoneal injection once per day. The rats in the control and HHcy groups were intraperitoneally administered the same amount of double-distilled water. The above experimental protocol was maintained for 6 weeks prior to examination.

The body weight and systolic blood pressure/diastolic blood pressure (SBP/DBP) of conscious rats were monitored weekly (16). At the end of the experiment in week 6 , blood samples were collected from each rat by cardiac puncture using an EDTA-treated syringe following anesthesia. Serum was prepared by centrifugation at $3,000 \mathrm{x}$ g for $20 \mathrm{~min}$ at $4^{\circ} \mathrm{C}$ and then stored at $-80^{\circ} \mathrm{C}$ until assayed. The aorta was extracted from the animal immediately following sacrifice. A section of the abdominal aorta was kept at $-71^{\circ} \mathrm{C}$ and chopped into small sections, homogenized in PBS on ice and then centrifuged at $1,000 \mathrm{x}$ g for $10 \mathrm{~min}$ prior to performing a biochemical analysis The remainder was frozen and prepared with a $10-\mu \mathrm{m}$ thickness using a cryostat microtome for reactive oxygen species (ROS) measurement. In order to analyze the isometric force, the surrounding fat and connective tissues of the thoracic aorta were carefully dissociated. The study protocol was approved by the Animal Care and Use Committee of The First Affiliated Hospital, College of Medicine, Zhejiang University. Ethical approval for the present study was obtained from the Ethics Committee of the First Affiliated Hospital of the College of Medicine of Zhejiang University.

Determination of total serum Hcy, glucose and cholesterol levels. Total serum glucose and cholesterol levels were determined by standard enzymatic techniques (17). Total serum Hcy concentrations were measured using a commercially available ELISA kit (CSB-E13376r; Cusabio Biotech Co., Ltd., College Park, MD, USA).
Determination of biochemical parameters in aortic homogenates. The abdominal aorta was used to measure ROS, catalase, superoxide dismutase (SOD), endothelial NOS (eNOS), NO and matrix metalloproteinases (MMPs). The protein content was accessed in an aliquot of the homogenate by the bicinchoninic acid assay method (Nanjing Jiancheng Bioengineering Research Institute, Nanjing, China).

Measurement of ROS levels. Aortic tissue was prepared as previously described $(18,19)$. The fluorescent probes 2,7-dichlorofluorescin-diacetate (DCFH-DA) and dihydroethidium (DHE) (both from Molecular Probes, Inc., Eugene, OR, USA) were used to evaluate the amount of hydrogen peroxide and superoxide anion, respectively. In the cytoplasm, the ester groups of DCFH-DA are hydrolyzed by cellular esterase to form DCFH, which is subsequently oxidized by intracellular ROS to highly fluorescent DCF, whereas DHE reacts with ROS and forms ethidium bromide, which binds to DNA and produces red fluorescence. The fluorescence (excitation $488 \mathrm{~nm}$ and emission $525 \mathrm{~nm}$; excitation $518 \mathrm{~nm}$ and emission $605 \mathrm{~nm}$ ) was monitored using a fluorescence microscope (Nikon TE2000; Nikon Corporation, Tokyo, Japan). The fluorescence intensities were recorded and analyzed using a charge-coupled device camera (CoolSNAP HQ) with an image analysis system (MetaMorph) (both from Nippon Roper K.K., Chiba, Japan).

Measurement of catalase and SOD activities. The activities of catalase (catalase assay kit; cat no. A007-1) and SOD (superoxide dismutase assay kit; cat no. A001-3) were determined using commercially available kits following the manufacturer's instructions (Nanjing Jiancheng Bioengineering Research Institute). The catalase activity assay was based on the reaction of methanol with the enzyme in the presence of an optimal concentration of hydrogen peroxide, while the SOD activity assay utilized xanthine oxidase and hypoxanthine to generate superoxide radicals.

Measurement of the levels of NO and the activity of eNOS. NO is rapidly consumed to generate peroxynitrite in the presence of superoxide (20). Therefore, aortic nitrite levels were measured as a level of NO inactivation due to superoxide. Nitrite was estimated colorimetrically using Griess reagent (21). The activity of eNOS was determined from the rate of conversion of L-arginine to L-citrulline. All assays were performed according to the manufacturer's instructions (NO assay kit; cat no. A012; NOS typed assay kit; cat no. A014-1; Nanjing Jiancheng Bioengineering Research Institute).

Measurement of the concentrations of MMP-2 and -9. MMP-2 and -9 were assessed using commercial ELISA kits (Rat MMP-2 ELISA kit, cat no. RA20502; Rat MMP-9 ELISA kit, cat no. RA20128; BioSwamp, Wuhan, China) according to the manufacturer's instructions (22).

Measurement of vasodilation using isometric tension recordings. Prepared thoracic aortic rings were suspended isometrically between two stirrups in an organ bath filled with $10 \mathrm{ml}$ modified Krebs solution at $37^{\circ} \mathrm{C}$, which was ventilated continuously with $95 \% \mathrm{O}_{2}$ and $5 \% \mathrm{CO}_{2}$. The isometric tension 
Table I. Body weight, blood pressure and metabolic parameters.

\begin{tabular}{lcccccc}
\hline Group & Body weight $(\mathrm{g})$ & SBP $(\mathrm{mmHg})$ & DBP $(\mathrm{mmHg})$ & Hcy $(\mu \mathrm{mol} / \mathrm{l})$ & Cholesterol $(\mathrm{mmol} / \mathrm{l})$ & Glucose $(\mathrm{mmol} / \mathrm{l})$ \\
\hline Control & $450.8 \pm 15.0$ & $103.08 \pm 3.60$ & $65.33 \pm 2.71$ & $6.92 \pm 0.61$ & $0.85 \pm 0.11$ & $4.96 \pm 0.32$ \\
HHcy & $448.0 \pm 13.8$ & $115.91 \pm 3.70^{\mathrm{a}}$ & $81.83 \pm 2.86^{\mathrm{a}}$ & $34.78 \pm 1.39^{\mathrm{a}}$ & $1.03 \pm 0.17^{\mathrm{a}}$ & $4.86 \pm 0.32$ \\
TEA & $449.7 \pm 13.5$ & $108.50 \pm 3.75^{\mathrm{b}}$ & $74.83 \pm 2.92^{\mathrm{b}}$ & $33.91 \pm 1.69^{\mathrm{b}}$ & $1.00 \pm 0.17^{\mathrm{c}}$ & $4.93 \pm 0.34$ \\
ASP & $452.2 \pm 13.9$ & $109.42 \pm 4.29^{\mathrm{b}}$ & $75.00 \pm 3.02^{\mathrm{b}}$ & $33.78 \pm 1.74^{\mathrm{a}}$ & $1.03 \pm 0.17^{\mathrm{a}}$ & $4.87 \pm 0.35$ \\
APS & $449.3 \pm 14.7$ & $114.92 \pm 4.34^{\mathrm{a}}$ & $79.83 \pm 2.69^{\mathrm{a}}$ & $33.93 \pm 2.00^{\mathrm{a}}$ & $1.04 \pm 0.16^{\mathrm{a}}$ & $4.86 \pm 0.30$ \\
ATF & $444.9 \pm 13.8$ & $115.18 \pm 2.86^{\mathrm{a}}$ & $80.58 \pm 2.94^{\mathrm{a}}$ & $34.41 \pm 2.32^{\mathrm{a}}$ & $1.03 \pm 0.17^{\mathrm{a}}$ & $4.97 \pm 0.32$ \\
\hline
\end{tabular}

SBP, systolic blood pressure; DBP, diastolic blood pressure; Hcy, homocysteine; HHcy, hyperhomocysteinemia; TEA, total extract of Astragalus membranaceus; ASP, Astragalus saponins; APS, Astragalus polysaccharides; ATF, Astragalus total flavonoids. ${ }^{\mathrm{a}} \mathrm{P}<0.01$ and ${ }^{\mathrm{c}} \mathrm{P}<0.05$ vs. the control group; ${ }^{\mathrm{P}}<0.01$ vs. the HHcy group.

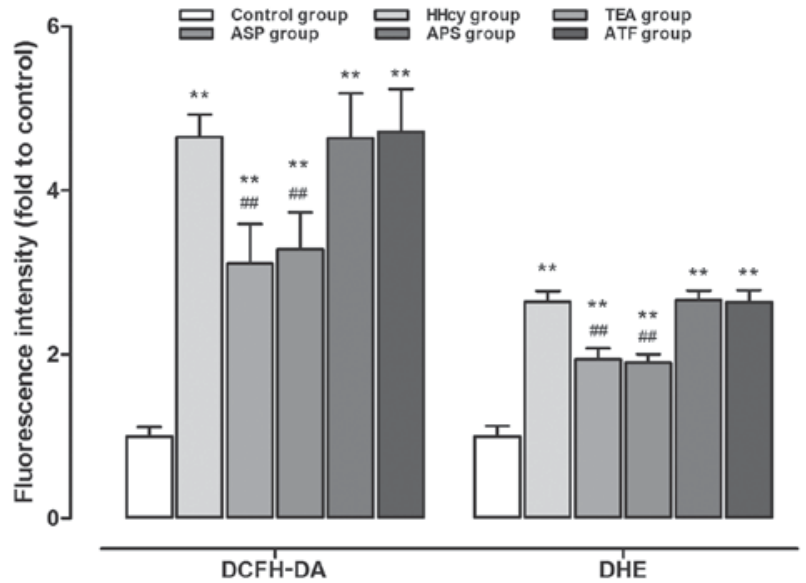

Figure 1. Hydrogen peroxide and superoxide anion accumulation in aortas as detected by DCFH-DA and DHE staining. ${ }^{* *} \mathrm{P}<0.01$ vs. the control group; ${ }^{\# \#} \mathrm{P}<0.01$ vs. the HHcy group. DCFH-DA, 2,7-dichlorofluorescein-diacetate; DHE, dihydroethidium; HHcy, hyperhomocysteinemia; TEA, total extract of Astragalus membranaceus; ASP, Astragalus saponins; APS, Astragalus polysaccharides; ATF, Astragalus total flavonoids.

was recorded using a force transducer (JZ101; metrical range, 0-5.0 g) and MedLab 5.0v recording system (Nanjing Medease Science and Technology Co., Ltd., Nanjing, China). A resting tension of $2.0 \mathrm{~g}$ was maintained for each ring. All rings were allowed to equilibrate for $60 \mathrm{~min}$ prior to the start of the experiment and then challenged twice by $\mathrm{KCl}(60 \mathrm{mmol} / \mathrm{l})$ to ensure the repeatability of contractions. Thereafter, phenylephrine (PE; $1 \mu \mathrm{mol} / \mathrm{l}$ ) was used to induce a steady contraction. Once a stable tension was established, vessel relaxation was determined as the response to an accumulative addition of an endothelium-dependent dilator agent, acetylcholine (Ach; $0.1 \mathrm{nmol} / 1$ to $1 \mu \mathrm{mol} / \mathrm{l}$ ), and an endothelium-independent dilator agent and sodium nitroprusside (SNP; $0.1 \mathrm{nmol} / 1$ to $1 \mu \mathrm{mol} / \mathrm{l})$. A number of vessels were incubated for $30 \mathrm{~min}$ with $0.1 \mathrm{mmol} / 1 \mathrm{~N}(\omega)$-nitro-L-arginine-methyl ester (L-NAME), an inhibitor of NOS, and the responses to Ach $(0.1 \mathrm{nmol} / \mathrm{l}$ to $1 \mu \mathrm{mol} / \mathrm{l})$ were then assessed.

Statistical analysis. All values are presented as the mean \pm standard deviation of $n$ experiments from different rats. Vasorelaxation is expressed as the percentage of the tension evoked by PE $(1 \mu \mathrm{mol} / \mathrm{l})$. The data was analyzed using GraphPad Prism software (GraphPad Software, Inc., La Jolla, CA, USA). Statistical analysis was performed by Student-Newman-Keuls test or one-way analysis of variance. $\mathrm{P}<0.05$ was considered to be statistically significant.

\section{Results}

Body weight, blood pressure and metabolic parameters. At the end of the experiment, there was no significant difference observed in the body weight among any of the groups (data not shown).

As shown in Table I, the high-methionine diet significantly increased the SBP/DBP $(115.91 \pm 3.70 / 81.83 \pm 2.86 \mathrm{mmHg})$ in rats of the HHcy group compared with the control group $(103.08 \pm 3.60 / 65.33 \pm 2.71 \mathrm{mmHg})$. TEA $(108.50 \pm 3.75 / 74.83 \pm 2.92 \mathrm{mmHg})$ and ASP $(109.42 \pm 4.29 / 75.00 \pm 3.02 \mathrm{mmHg})$ but not APS $(114.92 \pm 4.34 / 79.83 \pm 2.69 \mathrm{mmHg})$ and ATF $(115.18 \pm 2.86 / 80.58 \pm 2.94 \mathrm{mmHg})$ significantly attenuated the HHcy-induced increase in SBP/DBP.

Serum Hcy levels in rats fed with high-methionine diets [group ( $\mu \mathrm{mol} / \mathrm{l})$ : HHcy $(34.78 \pm 1.39)$, TEA (33.91 \pm 1.69$)$, ASP (33.78 \pm 1.74$)$, APS $(33.93 \pm 2.00)$ and ATF (34.41 \pm 2.32$)]$ were significantly higher compared with those in rats fed with regular diets (control group, 6.92 $\pm 0.61 \mu \mathrm{mol} / \mathrm{l}$ ). Treatment with TEA, ASP, APS or ATF did not influence the levels of serum Hcy. Serum cholesterol levels $(1.03 \pm 0.17 \mu \mathrm{mol} / \mathrm{l})$ in rats with high-methionine diets were higher than those in the control group $(0.85 \pm 0.11 \mu \mathrm{mol} / 1)$. However, treatment with TEA $(1.00 \pm 0.17 \mu \mathrm{mol} / 1)$, ASP $(1.03 \pm 0.17 \mu \mathrm{mol} / 1)$, APS $(1.04 \pm 0.16 \mu \mathrm{mol} / \mathrm{l})$ or ATF $(1.03 \pm 0.17 \mu \mathrm{mol} / \mathrm{l})$ did not exhibit any significant difference compared with the HHcy group. Additionally, there were no significant differences in serum glucose levels among any of the groups (Table I).

Redox system biomarker levels. The aortic DCFH-DA fluorescence intensity, which reflects the hydrogen peroxide level, was elevated in the rat aorta of the HHcy group when compared with the control group. Treatment with TEA or ASP, but not APS nor ATF, significantly reduced the hydrogen peroxide level in HHcy rats. A similar response was observed in the determination of the arterial DHE fluorescence intensity, which reflects the superoxide anion level (Fig. 1). 


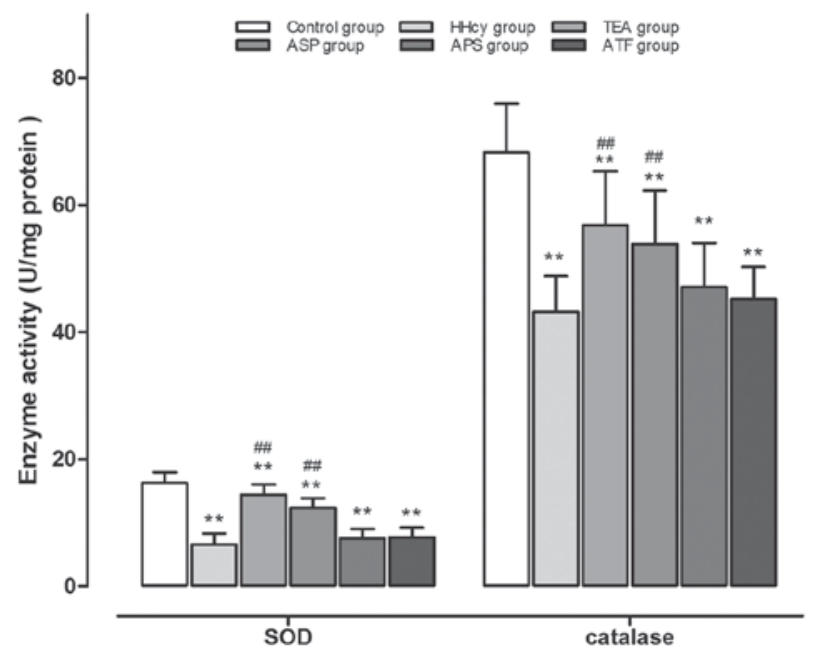

Figure 2. Activity of SOD and catalase in aortic homogenates. ${ }^{* *} \mathrm{P}<0.01$ vs. the control group; ${ }^{\# \#} \mathrm{P}<0.01$ vs. the HHcy group. SOD, superoxide dismutase; HHcy, hyperhomocysteinemia; TEA, total extract of Astragalus membranaceus; ASP, Astragalus saponins; APS, Astragalus polysaccharides; ATF, Astragalus total flavonoids.

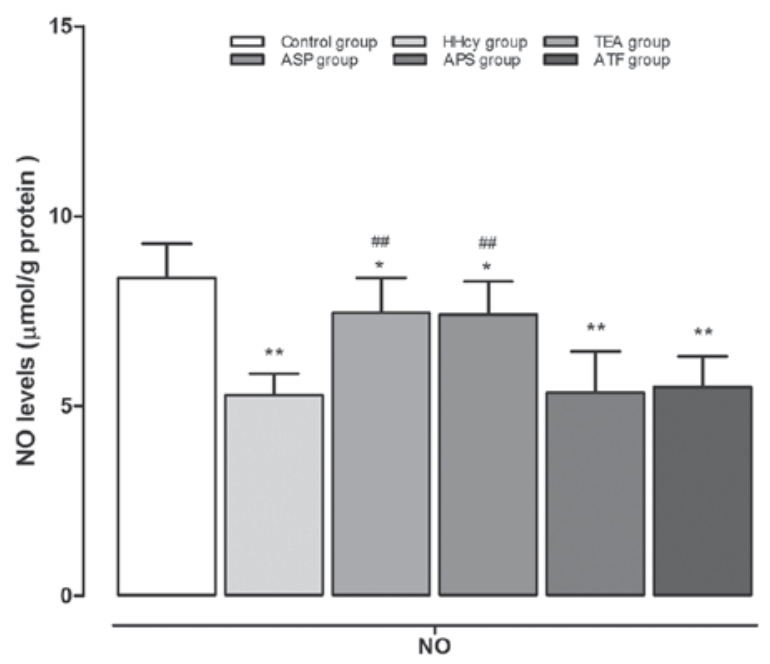

Figure 3. NO levels determined in aortic homogenates. ${ }^{* *} \mathrm{P}<0.01$ and ${ }^{*} \mathrm{P}<0.05$ vs. the control group; ${ }^{\# \#} \mathrm{P}<0.01$ vs. the HHcy group. NO, nitric oxide; HHcy, hyperhomocysteinemia; TEA, total extract of Astragalus membranaceus; ASP, Astragalus saponins; APS, Astragalus polysaccharides; ATF, Astragalus total flavonoids.

By contrast, the aortic SOD $(6.57 \pm 1.75 \mathrm{U} / \mathrm{mg}$ protein) and catalase $(43.17 \pm 5.62 \mathrm{U} / \mathrm{mg}$ protein) activities were evidently reduced in HHcy rats as compared with the control group (SOD, 16.30 $\pm 1.65 \mathrm{U} / \mathrm{mg}$ protein; catalase, 68.24 $\pm 7.76 \mathrm{U} / \mathrm{mg}$ protein, $\mathrm{P}<0.01)$. Additionally, TEA and ASP significantly increased the activities of SOD (TEA, 14.40 $1.62 \mathrm{U} / \mathrm{mg}$ protein; ASP, $12.29 \pm 1.58 \mathrm{U} / \mathrm{mg}$ protein) and catalase (TEA, $56.84 \pm 8.48 \mathrm{U} / \mathrm{mg}$ protein; ASP, $53.85 \pm 8.40 \mathrm{U} / \mathrm{mg}$ protein) compared with those in the HHcy group. The other components, APS and ATF, had no ameliorating effects on the activities of SOD and catalase (Fig. 2).

Levels of NO and the activity of eNOS. The aortic NO levels of various groups are shown in Fig. 3. The NO levels were significantly lower in the HHcy group $(5.29 \pm 0.56 \mu \mathrm{mol} / \mathrm{l} / \mathrm{g}$

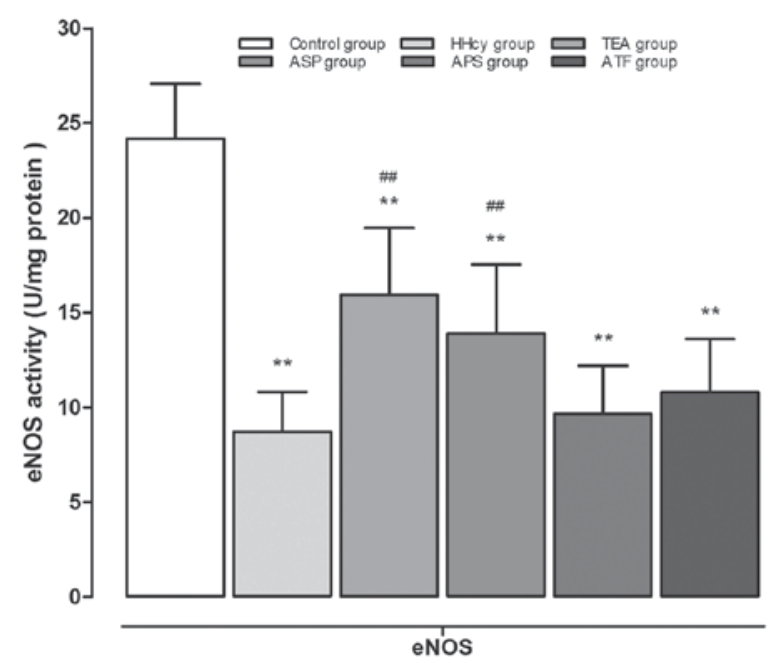

Figure 4. eNOS activity in aortic homogenates. ${ }^{* *} \mathrm{P}<0.01$ vs. the control group; ${ }^{\#} \mathrm{P}<0.01$ vs. the HHcy group. eNOS, endothelial nitric oxide synthase; HHcy, hyperhomocysteinemia; TEA, total extract of Astragalus membranaceus; ASP, Astragalus saponins; APS, Astragalus polysaccharides; ATF, Astragalus total flavonoids.

protein) compared with the control group $(8.37 \pm 0.90 \mu \mathrm{mol} / \mathrm{l} / \mathrm{g}$ protein). Furthermore, TEA $(7.45 \pm 0.92 \mu \mathrm{mol} / \mathrm{l} / \mathrm{g}$ protein) and ASP $(7.41 \pm 0.87 \mu \mathrm{mol} / \mathrm{l} / \mathrm{g}$ protein) treatment significantly increased aortic NO contents as compared with those in the HHcy group. Finally, there was no significant change in the aortic NO levels of the APS $(5.35 \pm 1.09 \mu \mathrm{mol} / \mathrm{l} / \mathrm{g}$ protein) and ATF $(5.50 \pm 0.81 \mu \mathrm{mol} / \mathrm{l} / \mathrm{g}$ protein) groups compared with the HHcy group.

As shown in Fig. 4, chronic exposure to Hcy decreased the eNOS activity by $63.9 \%$, which was markedly reversed by treatment with TEA and ASP, respectively. The eNOS activities in aortic homogenates from the APS and ATF groups demonstrated no difference in comparison with the HHcy group.

Tissue contents of MMP-2 and -9. When HHcy was successfully induced by a high-methionine diet, significantly increased MMP-2 (HHcy vs. control: 17.86 \pm 1.01 vs. $5.28 \pm 0.19 \mathrm{ng} / \mathrm{mg}$ protein) and MMP-9 (HHcy vs. control: $10.62 \pm 1.33$ vs. $2.93 \pm 0.43 \mathrm{ng} / \mathrm{mg}$ protein) concentrations were observed. The increase in MMP-2 content was clearly restrained by TEA $(10.32 \pm 1.21 \mathrm{ng} / \mathrm{mg}$ protein) or ASP $(11.03 \pm 0.97 \mathrm{ng} / \mathrm{mg}$ protein) compared with the HHcy group $(17.86 \pm 1.01 \mathrm{ng} / \mathrm{mg}$ protein). MMP-9 was also reduced to a similar extent by TEA or ASP. However, APS and ATF did not appear to influence the concentration of MMP-2 or -9 (Fig. 5).

Relaxation response of the thoracic aorta to Ach or SNP. In a dose-dependent manner, Ach $(0.1 \mathrm{nmol} / 1$ to $1 \mu \mathrm{mol} / \mathrm{l})$ elicited greater relaxations of the aortic rings from the control group compared with those from the HHcy group. Furthermore, TEA or ASP, but not APS or ATF, significantly increased the dilations of aortic rings compared with those in the HHcy group. However, the relaxation responses of aortic rings from TEA or ASP groups did not differ markedly from those of the HHcy group (Fig. 6).

Following incubation for $30 \mathrm{~min}$ with L-NAME, Ach-induced relaxation of aortic rings from the control and 


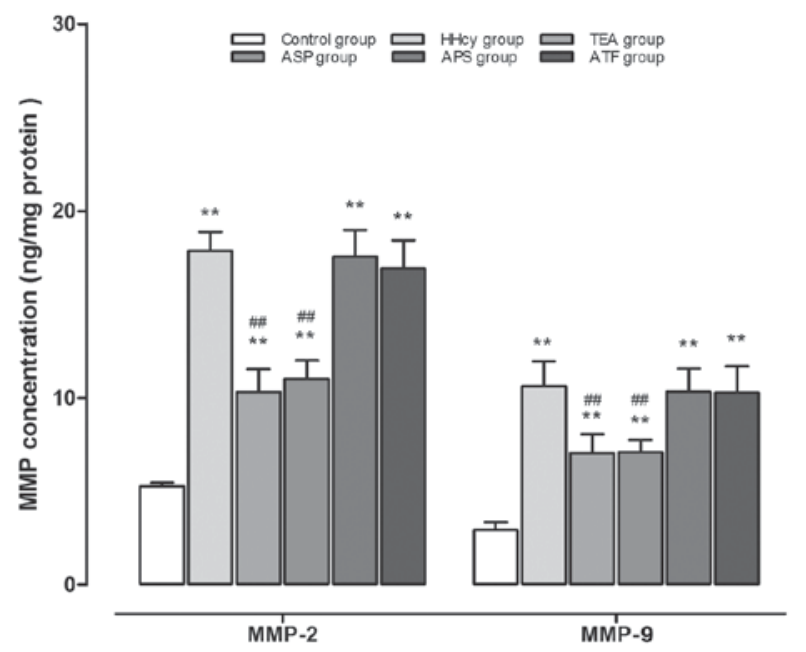

Figure 5. Tissue concentration of MMP-2 and -9 in aortic homogenates ${ }^{* *} \mathrm{P}<0.01$ vs. the control group; ${ }^{\# \#} \mathrm{P}<0.01$ vs. the HHcy group. MMP, matrix metalloproteinase; HHcy, hyperhomocysteinemia; TEA, total extract of Astragalus membranaceus; ASP, Astragalus saponins; APS, Astragalus polysaccharides; ATF, Astragalus total flavonoids.

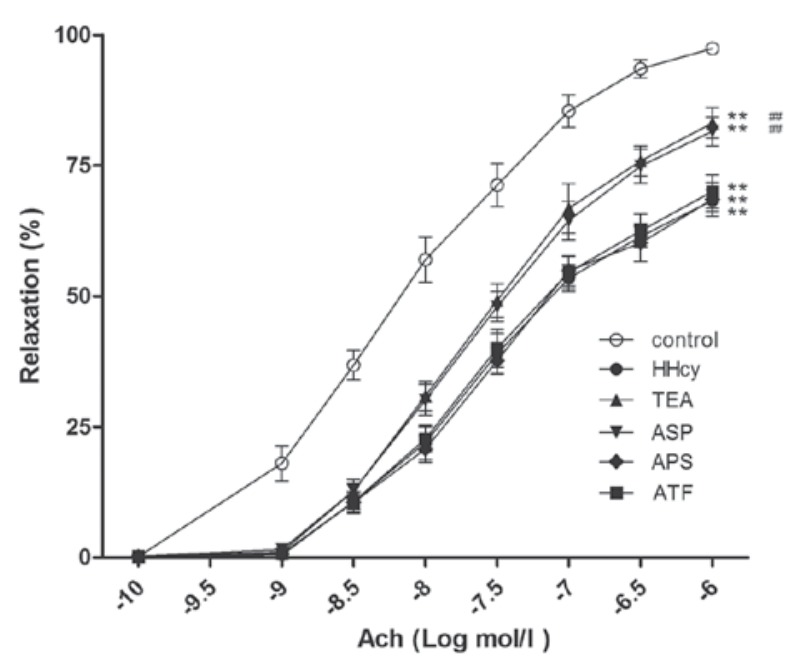

Figure 6. Endothelium-dependent relaxation in response to Ach. ${ }^{* *} \mathrm{P}<0.01$ vs. the control group; ${ }^{\# \#} \mathrm{P}<0.01$ vs. the HHcy group. Ach, acetylcholine; HHcy, hyperhomocysteinemia; TEA, total extract of Astragalus membranaceus; ASP, Astragalus saponins; APS, Astragalus polysaccharides; ATF, Astragalus total flavonoids.

TEA group, but not from the HHcy group, decreased significantly (Fig. 7). Furthermore, in a dose-dependent manner, SNP elicited similar dilations of aortic rings from the control, HHcy and TEA groups (Fig. 8).

\section{Discussion}

Epidemiological studies have revealed that moderate HHcy is highly prevalent in the general population and HHcy has been recognized as an increased risk factor for the development of cardiovascular disease (23).

The present study observed a sustained increase of the resting SBP/DBP and significantly elevated cholesterol levels when moderate HHcy was induced by chronic methionine feeding. These results were consistent with previous clinical

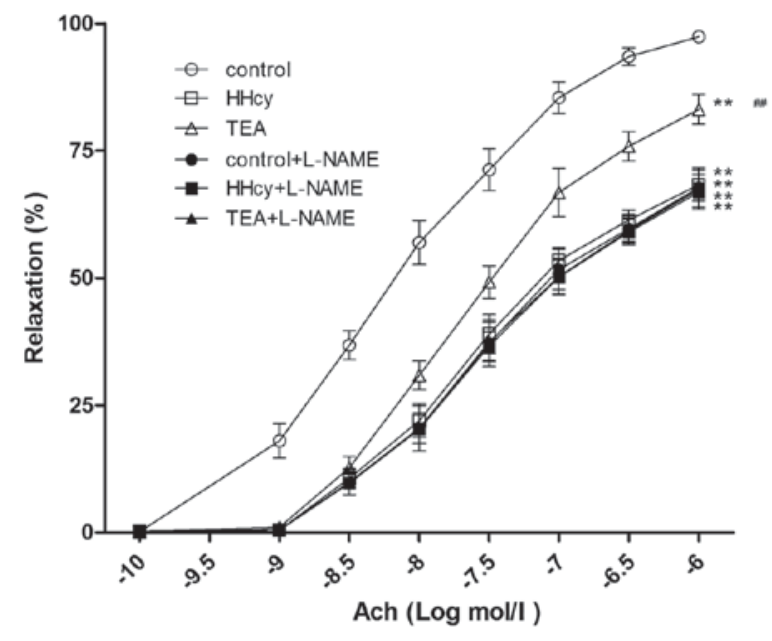

Figure 7. Vessel relaxation response to Ach in the presence or absence of L-NAME ${ }^{* *} \mathrm{P}<0.01$ vs. the control group; ${ }^{\#} \mathrm{P}<0.01$ vs. the HHcy group. Ach, acetylcholine; L-NAME, N( $\omega)$-nitro-L-arginine-methylester; HHcy, hyperhomocysteinemia; TEA, total extract of Astragalus membranaceus.

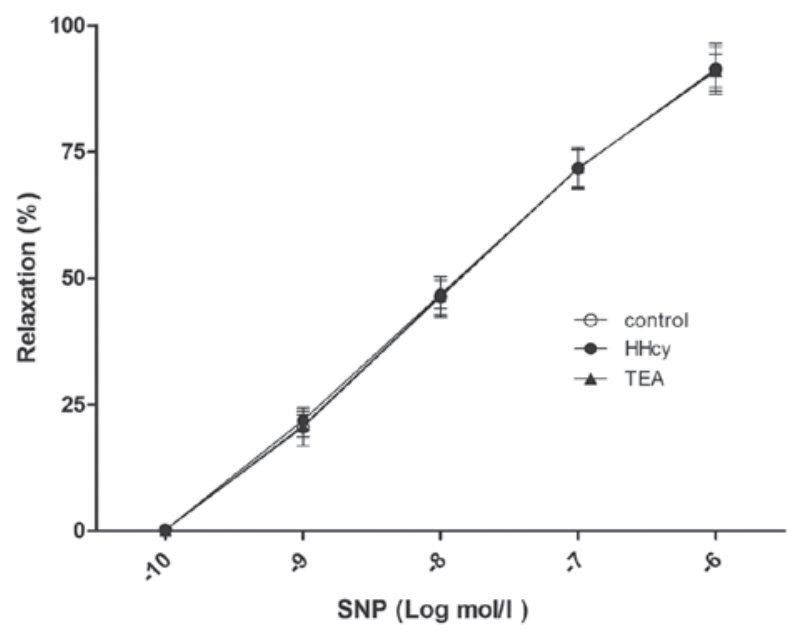

Figure 8. Endothelium-independent relaxation in response to SNP. SNP, sodium nitroprusside; HHcy, hyperhomocysteinemia; TEA, total extract of Astragalus membranaceus.

and animal studies $(24,25)$. Hcy levels have been reported to be higher in patients with essential hypertension compared with normotensive subjects, with higher levels of Hcy being associated with an increased risk of hypertension (26). Furthermore, increased SBP/DBP returned to control values when Hcy levels were normalized following the discontinuation of Hcy-elevating treatment (27). Additionally, the accumulation of lipids in the arterial wall, particularly cholesterol, is a major event preceding the formation of atherosclerotic lesions (28-30). Thus, it may be hypothesized that there is a causal correlation between HHcy and cardiovascular changes.

According to previous studies, one of the mechanisms by which HHcy impairs the vessel is Hcy-elicited endothelial dysfunction (5-7). Our previous in vitro studies $(13,14)$ and the present in vivo study indicated that Hcy impaired Ach-induced endothelium-dependent vasodilation, but did not alter SNP-induced endothelium-independent vasorelaxation. Additionally, pretreatment with L-NAME, an inhibitor of 
NOS, did not induce further relaxation of aortic rings from HHcy rats. Furthermore, it was demonstrated that chronic exposure to Hcy decreased the aortic NO content and inhibited eNOS activity. These results suggest that HHcy induced endothelial dysfunction by the endogenous NO/NOS pathway.

One of the mechanisms by which every component of the NO metabolism, including synthesis, release or action, may be impaired is an increased formation of ROS and the diminished expression and activity of antioxidant enzymes. The results of the present study indicated that hydrogen peroxide and superoxide anion were significantly accumulated in the aorta of the HHcy group compared with the control group, while the activities of SOD and catalase were evidently reduced.

An increase in oxidative stress accompanied by decreased NO bioavailability may lead to the increased activity of MMPs. MMPs, a family of specialized proteases, initiate the process of breakdown and synthesis of the extracellular matrix that is responsible for vascular remodeling (31). In the present study, an increased concentration of MMP-2 and -9 in the aorta of HHcy rats was also observed.

Thus, it has been unequivocally established that oxidative toxicity, the contribution of the endogenous NOS/NO system and MMP-mediated vascular remodeling are primary pathophysiological mechanisms underlying Hcy-mediated vascular dysfunction. However, western medicines for the cure of HHcy-evoked vascular disease are lacking.

AM has long been prescribed as a 'qi supplement' in traditional Chinese medicine (9). Experimental and clinical data have shown that AM and its constituents have cardiovascular protective effects, including the amelioration of atherosclerosis, induction of vasodilation and lowering of blood pressure $(32,33)$.

ASP is the most predominant of the bioactive compounds in the roots of AM. Meng et al (34) reported that ASP is able to increase free radical removal and decrease lipid peroxidation in isoproterenol-treated cardiomyocytes. Previous studies have indicated that astragaloside IV-induced improvements in vasorelaxation are associated with increased levels of aortic NO and cGMP $(11,13,14)$. In a study by Huang et al $(35)$, the results revealed that ASP protects against ischemia-reperfusion injury by modulation of MMP-9/tissue inhibitor of metalloproteinases 1 .

The present results demonstrate that TEA and its constituents have no influence on the serum levels of Hcy, cholesterol and glucose. TEA and ASP clearly controlled the HHcy-induced increase in SBP/DBP in rats. Furthermore, they decreased the levels of hydrogen peroxide and superoxide anion, increased the activities of SOD and catalase, restrained the concentrations of MMP-2 and -9, and increased the aortic NO contents and eNOS activities in aortic homogenates from HHcy rats. Finally, they significantly enhanced the endothelium-dependent vasorelaxation of aortic rings of HHcy rats.

It is worthy of note that although APS and ATF are considered the most important components of this plant and have been attributed with multiple biological activities $(9,32,33)$, ATF and APS were not observed to be as efficacious as TEA and ASP in the present study. Wang et al (36) reported that ATF benefits cardiovascular disease via its potent antioxidant activity and Zhang et al (37) indicated that APS reversed palmitate-induced cardiomyocyte toxicity partially through the suppression of ROS accumulation. However, the antioxidant effects of ATF and APS have been demonstrated to be significantly lower than those of ASP (38). Additionally, our previous in vitro study demonstrated that ASP, but not APS, protected endothelium-dependent relaxation against the acute injury from Hcy through NO regulatory pathways (14).

In conclusion, the present study demonstrated that TEA and its ASP constituents are able to improve vascular dysfunction and remodeling in HHcy, which may be attributed to its potent effects against oxidation, activity of the MMPs and endothelial dysfunction.

\section{Acknowledgements}

The present study was supported by the Zhejiang Province Administration of Traditional Chinese Medicine (grant nos. 2011ZA054 and 2014ZA065).

\section{References}

1. Steed MM and Tyagi SC: Mechanisms of cardiovascular remodeling in hyperhomocysteinemia. Antioxid Redox Signal 15: 1927-1943, 2011

2. Yusuf S, Reddy S, Ounpuu S and Anand S: Global burden of cardiovascular diseases: Part I: General considerations, the epidemiologic transition, risk factors, and impact of urbanization. Circulation 104: 2746-2753, 2001.

3. Veeranna V, Zalawadiya SK, Niraj A, Pradhan J, Ference B, Burack RC, Jacob S and Afonso L: Homocysteine and reclassification of cardiovascular disease risk. J Am Coll Cardiol 58: 1025-1033, 2011.

4. Jakubowski H, Perla-Kaján J, Finnell RH, Cabrera RM, Wang H, Gupta S, Kruger WD, Kraus JP and Shih DM: Genetic or nutritional disorders in homocysteine or folate metabolism increase protein N-homocysteinylation in mice. FASEB J 23: 1721-1727, 2009.

5. Tawakol A, Omland T, Gerhard M, Wu JT and Creager MA: Hyperhomocyst(e)inemia is associated with impaired endothelium-dependent vasodilation in humans. Circulation 95: 1119-1121, 1997.

6. Dayal S, Bottiglieri T, Arning E, Maeda N, Malinow MR, Sigmund CD, Heistad DD, Faraci FM and Lentz SR: Endothelial dysfunction and elevation of S-adenosylhomocysteine in cystathionine beta-synthase-deficient mice. Circ Res 88: 1203-1209, 2001.

7. Symons JD, Rutledge JC, Simonsen U and Pattathu RA: Vascular dysfunction produced by hyperhomocysteinemia is more severe in the presence of low folate. Am J Physiol Heart Circ Physiol 290: H181-H191, 2006.

8. Martí-Carvajal AJ, Solà I, Lathyris D, Karakitsiou DE and Simancas-Racines D: Homocysteine-lowering interventions for preventing cardiovascular events. Cochrane Database Syst Rev: CD006612, 2013.

9. No authors listed: Astragalus membranaceus. Monograph. Altern Med Rev 8: 72-77, 2003.

10. Ko JK, Lam FY and Cheung AP: Amelioration of experimental colitis by Astragalus membranaceus through anti-oxidation and inhibition of adhesion molecule synthesis. World $\mathrm{J}$ Gastroenterol 11: 5787-5794, 2005.

11. Zhang N, Wang XH, Mao SL and Zhao F: Astragaloside IV improves metabolic syndrome and endothelium dysfunction in fructose-fed rats. Molecules 16: 3896-3907, 2011.

12. Gui D, Guo Y, Wang F, Liu W, Chen J, Chen Y, Huang J and Wang N: Astragaloside IV, a novel antioxidant, prevents glucose-induced podocyte apoptosis in vitro and in vivo. PLoS One 7: e39824, 2012.

13. Qiu LH, Xie XJ and Zhang BQ: Astragaloside IV improves homocysteine-induced acute phase endothelial dysfunction via antioxidation. Biol Pharm Bull 33: 641-646, 2010.

14. Zhang BQ, Hu SJ, Qiu LH, Zhu JH, Xie XJ, Sun J, Zhu ZH, Xia Q and Bian K: Effects of Astragalus membranaceus and its main components on the acute phase endothelial dysfunction induced by homocysteine. Vascul Pharmacol 46: 278-285, 2007. 
15. Wang G, Woo CW, Sung FL, Siow YL and O K: Increased monocyte adhesion to aortic endothelium in rats with hyperhomocysteinemia: Role of chemokine and adhesion molecules. Arterioscler Thromb Vasc Biol 22: 1777-1783, 2002.

16. Yu Z, Xu Z, Li H, Chen J, Huang Z and Tang Y: Recognition and neutralization of angiotensins I and II using an artificial nanoge receptor fabricated by ligand specificity determinant imprinting. Chem Commun (Camb) 50: 2728-2731, 2014.

17. Ran JM, Lao GC, Xu G, Xie B, Zhang Y, Liu W, Feng Q and Guo J: Effects of angiotensin II type 1 receptor blocker on triglyceride metabolism in the liver: Experiment with Zucker fatty rats. Zhonghua Yi Xue Za Zhi 88: 1557-1561, 2008 (In Chinese).

18. Wu S, Gao X, Yang S, Meng M, Yang X and Ge B: The role of endoplasmic reticulum stress in endothelial dysfunction induced by homocysteine thiolactone. Fundam Clin Pharmacol 29: 252-259, 2015

19. Leung SB, Zhang H, Lau CW, Huang Y and Lin Z: Salidroside improves homocysteine-induced endothelial dysfunction by reducing oxidative stress. Evid Based Complement Alternat Med 2013: 679635, 2013.

20. Faraci FM: Hyperhomocysteinemia: A million ways to lose control. Arterioscler Thromb Vasc Biol 23: 371-373, 2003.

21. Majithiya JB, Paramar AN and Balaraman R: Pioglitazone, a PPAR-gamma agonist, restores endothelial function in aorta of streptozotocin-induced diabetic rats. Cardiovasc Res 66 $150-161,2005$

22. Hu Z, Wang Z, Wu H, Yang Z, Jiang W, Li L and Hu X: Ang II enhances noradrenaline release from sympathetic nerve endings thus contributing to the up-regulation of metalloprotease- 2 in aortic dissection patients' aorta wall. PLoS One 8: e76922, 2013

23. Hoogeveen EK, Kostense PJ, Beks PJ, Mackaay AJ, Jakobs C, Bouter LM, Heine RJ and Stehouwer CD: Hyperhomocysteinemia is associated with an increased risk of cardiovascular disease, especially in non-insulin-dependent diabetes mellitus: A population-based study. Arterioscler Thromb Vasc Biol 18: $133-138,1998$

24. de Bree A, van der Put NM, Mennen LI, Verschuren WM, Blom HJ, Galan P, Bates CJ, Herrmann W, Ullrich M, Dierkes J, et al: Prevalences of hyperhomocysteinemia, unfavorable cholesterol profile and hypertension in European populations. Eur J Clin Nutr 59: 480-488, 2005.

25. Fenyo IM, Florea IC, Raicu M and Manea A: Tyrphostin AG490 reduces NAPDH oxidase activity and expression in the aorta of hypercholesterolemic apolipoprotein E-deficient mice. Vascul Pharmacol 54: 100-106, 2011.

26. Jiang S, Hsu YH, Xu X, Xing H, Chen C, Niu T, Zhang Y, Peng S and Xu X: The C677T polymorphism of the methylenetetrahydrofolate reductase gene is associated with the level of decrease on diastolic blood pressure in essential hypertension patients treated by angiotensin-converting enzyme inhibitor. Thromb Res 113: 361-369, 2004.

27. Rodrigo R, Passalacqua W, Araya J, Orellana M and Rivera G: Homocysteine and essential hypertension. J Clin Pharmacol 43: 1299-1306, 2003.
28. Lim U and Cassano PA: Homocysteine and bloodpressure in the third national health and nutrition examination survey, 1988-1994. Am J Epidemiol 156: 1105-1113, 2002.

29. Resstel LB, de Andrade CR, Haddad R, Eberlin MN, deOliveiraAMand CorrêaFM: Hyperhomocysteinaemia-induced cardiovascular changes in rats. Clin Exp Pharmacol Physiol 35: 949-956, 2008

30. de Andrade CR, Tirapelli CR, Haddad R, Eberlin MN, Ramalho LN, Iyomasa MM, Uyemura SA and de Oliveira AM: Hyperhomocysteinemia induced by feeding rats diets rich in DL-homocysteine thiolactone promotes alterations on carotid reactivity independent of arterial structure. Vascul Pharmacol 51: 291-298, 2009.

31. Mujumdar VS, Aru GM and Tyagi SC: Induction of oxidative stress by homocyst(e)ine impairs endothelial function. J Cell Biochem 82: 491-500, 2001

32. Ren S, Zhang H, Mu Y, Sun M and Liu P: Pharmacological effects of Astragaloside IV: A literature review. J Tradit Chin Med 33: 413-416, 2013.

33. Ho JW and Jie M: Pharmacological activity of cardiovascular agents from herbal medicine. Cardiovasc Hematol Agents Med Chem 5: 273-277, 2007.

34. Meng D, Chen XJ, Bian YY, Li P, Yang D and Zhang JN: Effect of astragalosides on intracellular calcium overload in cultured cardiac myocytes of neonatal rats. Am J Chin Med 33: 11-20, 2005.

35. Huang X, Tan H, Chen B and Deng C: Influence of astragalosides and Panax notoginseng saponins compatibility on MMP-9 and TIMP-1 after cerebral ischemia reperfusion in mice. Zhong Zhong Yao Za Zhi 35: 2187-2191, 2010 (In Chinese).

36. Wang D, Zhuang Y, Tian Y, Thomas GN, Ying M and Tomlinson B: Study of the effects of total flavonoids of Astragalus on atherosclerosis formation and potential mechanisms. Oxid Med Cell Longev 2012: 282383, 2012.

37. Zhang J, Gu JY, Chen ZS, Xing KC and Sun B: Astragalus polysaccharide suppresses palmitate-induced apoptosis in human cardiac myocytes: The role of Nrf1 and antioxidant response. Int J Clin Exp Pathol 8: 2515-2524, 2015.

38. Liu CY, Gu ZL, Zhou WX and Guo CY: Effect of Astragalus complanatus flavonoid on anti-liver fibrosis in rats. World $\mathrm{J}$ Gastroenterol 11: 5782-5786, 2005. 